Pacific

Journal of

Mathematics

UNFAITHFUL COMPLEX HYPERBOLIC TRIANGLE GROUPS, III: ARITHMETICITY AND COMMENSURABILITY

JULIEN PAUPERT

Volume $245 \quad$ No. 2

April 2010 


\title{
UNFAITHFUL COMPLEX HYPERBOLIC TRIANGLE GROUPS, III: ARITHMETICITY AND COMMENSURABILITY
}

\author{
JULIEN PAUPERT
}

\begin{abstract}
We prove that the so-called sporadic complex reflection triangle groups in $\mathrm{SU}(2,1)$ are all nonarithmetic but one, and that they are not commensurable to Mostow or Picard lattices (with a small list of exceptions). This provides an infinite list of potential new nonarithmetic lattices in $\mathrm{SU}(2,1)$.
\end{abstract}

\section{Introduction}

Parker and Paupert [2009] considered symmetric triangle groups $\Delta$ in $\mathrm{SU}(2,1)$ generated by three complex reflections through angle $2 \pi / p$ for $p \geqslant 3$; the case of order 2 was studied in [Parker 2008b]. By "symmetric", we mean that the group in question is generated by three complex reflections $R_{1}, R_{2}$ and $R_{3}$ with the property that there exists an isometry $J$ of order 3 such that $R_{j+1}=J R_{j} J^{-1}$, where $j$ is taken mod 3. We study the group $\Gamma$ generated by $R_{1}$ and $J$, which contains $\Delta$ with index 1 or 3.

This type of group was first studied by Mostow [1980] for $p=3,4,5$, where an additional condition was imposed on the $R_{j}$, namely the braid relation $R_{i} R_{j} R_{i}=$ $R_{j} R_{i} R_{j}$; these provided the first examples of nonarithmetic lattices in $\mathrm{SU}(2,1)$. Further nonarithmetic lattices in $\mathrm{SU}(n, 1)$ for $n \leqslant 9$ were constructed in [Deligne and Mostow 1986] and [Mostow 1986] as monodromy groups of certain hypergeometric functions (the lattices from the former, in dimension 2, were known to Picard, who did not consider their arithmetic nature). These lattices are (commensurable with) groups generated by complex reflections $R_{j}$ with other values of $p$ [Mostow 1986; Sauter 1990]. Subsequently no new nonarithmetic lattices have been constructed.

In [Parker and Paupert 2009], we showed that symmetric complex reflection triangle groups $\Delta=\left\langle R_{1}, R_{2}, R_{3}\right\rangle$, if they are discrete and if $R_{1} R_{2}$ and $R_{1} R_{2} R_{3}$ are elliptic, come in three flavors: Mostow's lattices, subgroups thereof, and a third class, which we called sporadic groups (see Section 2 for a precise definition). Our main motivation is that these new groups are candidates for nonarithmetic lattices in $\operatorname{SU}(2,1)$. In this paper we analyze the adjoint trace fields $\mathbb{Q}[\operatorname{Tr} \operatorname{Ad} \Gamma]$

MSC2000: 20H10, 22E40, 51M10.

Keywords: nonarithmetic lattices, complex reflection groups, complex hyperbolic geometry. 
of the sporadic groups $\Gamma$, and use this to determine which sporadic groups are arithmetic, and which ones are commensurable to Mostow or Picard lattices. The main results are Theorems 4.1 and 5.2, which say in essence that all sporadic groups are nonarithmetic, except one that was studied in [Parker and Paupert 2009], and moreover that they are not commensurable to any of the Mostow or Picard lattices, with an explicit list of possible exceptions.

The only required notions of complex hyperbolic geometry are the definitions of elliptic and regular elliptic isometries, as well as complex reflections. These are standard and can be found for instance in the book [Goldman 1999].

\section{Sporadic groups}

We recall the setup and main results from [Parker and Paupert 2009]. Our starting point was that groups $\Gamma=\left\langle R_{1}, J\right\rangle$ as defined above can be parametrized up to conjugacy by $\tau=\operatorname{Tr}\left(R_{1} J\right)$; we denoted by $\Gamma(\psi, \tau)$ the group generated by a complex reflection $R_{1}$ through angle $\psi$ and a regular elliptic isometry $J$ of order 3 such that $\tau=\operatorname{Tr}\left(R_{1} J\right)$. The generators for this group were given in the form

$$
\begin{aligned}
J & =\left[\begin{array}{lll}
0 & 0 & 1 \\
1 & 0 & 0 \\
0 & 1 & 0
\end{array}\right], \\
R_{1} & =\left[\begin{array}{ccc}
e^{2 i \psi / 3} & \tau & -e^{i \psi / 3} \bar{\tau} \\
0 & e^{-i \psi / 3} & 0 \\
0 & 0 & e^{-i \psi / 3}
\end{array}\right] .
\end{aligned}
$$

These preserve the Hermitian form $\langle\mathbf{z}, \mathbf{w}\rangle=\mathbf{w}^{*} H_{\tau} \mathbf{z}$ where

$$
H_{\tau}=\left[\begin{array}{ccc}
2 \sin (\psi / 2) & -i e^{-i \psi / 6} \tau & i e^{i \psi / 6} \bar{\tau} \\
i e^{i \psi / 6} \bar{\tau} & 2 \sin (\psi / 2) & -i e^{-i \psi / 6} \tau \\
-i e^{-i \psi / 6} \tau & i e^{i \psi / 6} \bar{\tau} & 2 \sin (\psi / 2)
\end{array}\right] .
$$

This always produces a subgroup $\Gamma$ of GL $(3, \mathbb{C})$, but the signature of $H_{\tau}$ depends on the values of $\psi$ and $\tau$. We determined the corresponding parameter space for $\tau$ for any fixed value of $\psi$ [Parker and Paupert 2009, Sections 2.4 and 2.6]. When $\Gamma$ preserves a Hermitian form of signature $(2,1)$, we will say that $\Gamma$ is hyperbolic.

We found necessary conditions for these groups to be discrete, and these conditions produced, along with the groups previously studied by Mostow [1980], a list of possibly discrete such groups:

Theorem 2.1. Let $R_{1}$ be a complex reflection of order $p$ and $J$ a regular elliptic isometry of order 3 in $\mathrm{PU}(2,1)$. Suppose that $R_{1} J$ and $R_{1} R_{2}=R_{1} J R_{1} J^{-1}$ are elliptic. If the group $\Gamma=\left\langle R_{1}, J\right\rangle$ is discrete then one of the following is true:

- $\Gamma$ is one of Mostow's lattices. 
- $\Gamma$ is a subgroup of one of Mostow's lattices.

- $\Gamma$ is one of the sporadic groups listed below.

Mostow's lattices correspond to $\tau=e^{i \phi}$ for some angle $\phi$, while subgroups of Mostow's lattices correspond to $\tau=e^{2 i \phi}+e^{-i \phi}$ for some angle $\phi$, and sporadic groups (this can be taken as a definition) are those for which $\tau$ takes one of the 18 values $\left\{\sigma_{1}, \bar{\sigma}_{1}, \ldots, \sigma_{9}, \bar{\sigma}_{9}\right\}$ where the $\sigma_{i}$ are given in the following list:

$$
\begin{aligned}
& \sigma_{1}:=e^{i \pi / 3}+e^{-i \pi / 6} 2 \cos (\pi / 4), \quad \sigma_{4}:=e^{2 \pi i / 7}+e^{4 \pi i / 7}+e^{8 \pi i / 7}, \\
& \sigma_{2}:=e^{i \pi / 3}+e^{-i \pi / 6} 2 \cos (\pi / 5), \quad \sigma_{5}:=e^{2 \pi i / 9}+e^{-i \pi / 9} 2 \cos (2 \pi / 5), \\
& \sigma_{3}:=e^{i \pi / 3}+e^{-i \pi / 6} 2 \cos (2 \pi / 5), \quad \sigma_{6}:=e^{2 \pi i / 9}+e^{-\pi i / 9} 2 \cos (4 \pi / 5), \\
& \sigma_{7}:=e^{2 \pi i / 9}+e^{-i \pi / 9} 2 \cos (2 \pi / 7), \\
& \sigma_{8}:=e^{2 \pi i / 9}+e^{-i \pi / 9} 2 \cos (4 \pi / 7), \\
& \sigma_{9}:=e^{2 \pi i / 9}+e^{-i \pi / 9} 2 \cos (6 \pi / 7) .
\end{aligned}
$$

Therefore, for each value of $p \geqslant 3$, we have a finite number of new groups to study, the $\Gamma\left(2 \pi / p, \sigma_{i}\right)$ and $\Gamma\left(2 \pi / p, \bar{\sigma}_{i}\right)$, which are hyperbolic. We determined which sporadic groups are hyperbolic and listed them in the table in [Parker and Paupert 2009, Section 3.3]. Notably such groups exist for all values of $p$, and more precisely:

Proposition 2.1. For $p \geqslant 4$ and $\tau=\sigma_{1}, \sigma_{2}, \sigma_{3}, \bar{\sigma}_{4}, \sigma_{5}, \sigma_{6}, \sigma_{7}, \bar{\sigma}_{8}$ or $\sigma_{9}$, the groups $\Gamma(2 \pi / p, \tau)$ are hyperbolic.

When we study the question of arithmeticity of these groups, we will use the list of all hyperbolic sporadic groups, as well as the following normalization of the entries of our matrices:

Proposition 2.2 [Parker and Paupert 2009, Proposition 2.8]. The maps $R_{1}, R_{2}$ and $R_{3}$ may be conjugated within $\mathrm{SU}(2,1)$ and scaled so that their matrix entries lie in the ring $\mathbb{Z}\left[\tau, \bar{\tau}, e^{ \pm i \psi}\right]$.

Explicitly, we conjugate the previous matrices by $C=\operatorname{diag}\left(e^{-i \psi / 3}, 1, e^{i \psi / 3}\right)$ and rescale by $e^{-i \psi / 3}$. Conjugating by $C$ and rescaling by $2 \sin (\psi / 2)$ also brings $H_{\tau}$ to a Hermitian matrix with entries in the same ring $R=\mathbb{Z}\left[\tau, \bar{\tau}, e^{ \pm i \psi}\right]$. Therefore, a hyperbolic $\Gamma(\psi, \tau)$ can be realized as a subgroup of $\mathrm{SU}(H, R)$ where $H$ is an $R$-defined Hermitian form of signature $(2,1)$.

Finally, we showed that some of the hyperbolic sporadic groups are nondiscrete [Parker and Paupert 2009, Corollary 4.2, Proposition 4.5 and Corollary 6.4]:

Proposition 2.3. For $p \geqslant 3$ and ( $\tau$ or $\bar{\tau}=\sigma_{3}, \sigma_{8}$ or $\left.\sigma_{9}\right), \Gamma(2 \pi / p, \tau)$ is not discrete. Also, for $p \geqslant 3$ with $p \neq 5$ and $\left(\tau\right.$ or $\left.\bar{\tau}=\sigma_{6}\right), \Gamma(2 \pi / p, \tau)$ is not discrete. 


\section{Trace fields}

The trace field $\mathbb{Q}[\operatorname{Tr} \Gamma]$ is a classical invariant for a finitely generated subgroup $\Gamma$ of a linear group $G$. It is invariant under conjugacy, but not commensurability. (We will say that two subgroups $\Gamma_{1}$ and $\Gamma_{2}$ of $G$ are commensurable if there exists $g \in G$ such that $\Gamma_{1} \cap g \Gamma_{2} g^{-1}$ has finite index in both $\Gamma_{1}$ and $g \Gamma_{2} g^{-1}$ ). To obtain a commensurability invariant for such $\Gamma$, one can consider the trace field $\mathbb{Q}\left[\operatorname{Tr} \Gamma^{(n)}\right]$ (where $\Gamma^{(n)}$ is the subgroup of $\Gamma$ generated by $n$-th powers for $\Gamma \subset \operatorname{GL}(n, \mathbb{C})$ ), as in [Maclachlan and Reid 2003] for SL(2, $\mathbb{C})$ or as in [McReynolds 2006] for $\mathrm{SU}(2,1)$. Another possibility is the adjoint trace field $\mathbb{Q}[\operatorname{Tr} A d \Gamma]$, given by the adjoint representation Ad: $G \rightarrow \mathrm{GL}(\mathfrak{g})$, as in [Mostow 1980; 1986; Deligne and Mostow 1986] for $\mathrm{SU}(n, 1)$. The following result can be found for instance as [Deligne and Mostow 1986, Proposition 12.2.1]:

Proposition 3.1. $\mathbb{Q}[\operatorname{Tr} \mathrm{Ad} \Gamma]$ is a commensurability invariant.

This is the field that we will use here, as it is more convenient for our purposes. Indeed, this invariant trace field has been computed for all known nonarithmetic lattices in SU(2, 1). See the lists on [Mostow 1980, page 251] and [Deligne and Mostow 1986, page 86]. Moreover it is easy to compute (or at least estimate) by the following result:

Proposition 3.2. $\operatorname{Tr} \operatorname{Ad}(\gamma)=|\operatorname{Tr}(\gamma)|^{2}$ for $\gamma \in \mathrm{SU}(2,1)$,

This result is used several times in [Mostow 1980], where it is referred to as Lemma 4.2, but unfortunately its statement is missing from final edition.

Proof. If $U$ is a unitary group (of any signature), the adjoint representation of $U$ is isomorphic to the representation $U \otimes \bar{U}$.

We use this to find the following bounds for $\mathbb{Q}[\operatorname{Tr} \operatorname{Ad} \Gamma(\psi, \tau)]$ :

\section{Proposition 3.3.}

$\mathbb{Q}\left[\cos \psi,|\tau|^{2}, \operatorname{Re} \tau^{3}, \operatorname{Re}\left(e^{-i \psi} \tau^{3}\right)\right] \subset \mathbb{Q}[\operatorname{Tr} \operatorname{Ad} \Gamma(\psi, \tau)] \subset \mathbb{Q}\left[\tau, \bar{\tau}, e^{i \psi}\right] \cap \mathbb{R}$.

Proof. The second inclusion follows from Propositions 2.2 and 3.2. For the first inclusion, we use Proposition 3.2 and compute $|\operatorname{Tr}(\gamma)|^{2}$ for various words $\gamma$, using the table of traces from [Parker and Paupert 2009, Section 4.1]; see also formulae in [Pratoussevitch 2005]. We have

$$
\begin{aligned}
\left|\operatorname{Tr} R_{1}\right|^{2} & =5+4 \cos \psi, \\
\left|\operatorname{Tr} R_{1} J\right|^{2} & \left.=|\tau|^{2} \quad \text { (by the definition of } \tau\right), \\
\left|\operatorname{Tr}\left(R_{1} J\right)^{2}\right|^{2} & =|\tau|^{4}+4|\tau|^{2}-4 \operatorname{Re} \tau^{3}, \\
\left|\operatorname{Tr}\left(J^{-1} R_{1}\right)^{2}\right|^{2} & =|\tau|^{4}+4|\tau|^{2}-4 \operatorname{Re}\left(e^{-i \psi} \tau^{3}\right) .
\end{aligned}
$$


We list the corresponding elements of $\mathbb{Q}\left[\operatorname{Tr} \operatorname{Ad} \Gamma\left(2 \pi / p, \sigma_{i}\right)\right]$ in the table below. Numbers in the last three columns are not the values of $|\tau|^{2}, \operatorname{Re} \tau^{3}$ or $\operatorname{Re}\left(e^{-i \psi} \tau^{3}\right)$, but rather new algebraic numbers added to $\mathbb{Q}\left[\operatorname{Tr} \operatorname{Ad} \Gamma\left(2 \pi / p, \sigma_{i}\right)\right]$ by these values. For example, the first four zeros in the fourth column indicate that the corresponding $\operatorname{Re} \tau^{3}$ is already in $\mathbb{Q}\left[\cos \psi,|\tau|^{2}\right]$.

\begin{tabular}{l|cccr} 
& $\cos \psi$ & $|\tau|^{2}$ & $\operatorname{Re} \tau^{3}$ & $\operatorname{Re}\left(e^{-i \psi} \tau^{3}\right)$ \\
\hline$\sigma_{1}$ & $\cos 2 \pi / p$ & 0 & 0 & $\sqrt{2} \sin 2 \pi / p$ \\
$\sigma_{2}$ & $\cos 2 \pi / p$ & $\cos \pi / 5$ & 0 & $\sin 2 \pi / p$ \\
$\sigma_{3}$ & $\cos 2 \pi / p$ & $\cos 3 \pi / 5$ & 0 & $\sin 2 \pi / p$ \\
$\sigma_{4}$ & $\cos 2 \pi / p$ & 0 & 0 & $\sqrt{7} \sin 2 \pi / p$ \\
$\sigma_{5}$ & $\cos 2 \pi / p$ & 0 & $\cos 2 \pi / 5$ & $\sqrt{3} \sin 2 \pi / p$ \\
$\sigma_{6}$ & $\cos 2 \pi / p$ & 0 & $\cos 4 \pi / 5$ & $\sqrt{3} \sin 2 \pi / p$ \\
$\sigma_{7}$ & $\cos 2 \pi / p$ & $\cos \pi / 7$ & 0 & $\sqrt{3} \sin 2 \pi / p$
\end{tabular}

\section{Arithmeticity}

In [Parker and Paupert 2009, Propositions 6.5 and 6.6], we proved that only one of the sporadic groups with $p=3$, namely $\Gamma\left(2 \pi / 3, \bar{\sigma}_{4}\right)$, is contained in an arithmetic lattice in $\mathrm{SU}(2,1)$. (It was shown in [Parker 2008b] that all the corresponding groups with $p=2$ are arithmetic.) In this section we extend this to higher values of $p$, and show that in fact this group is the only such example among all higherorder sporadic groups:

Theorem 4.1. For $p \geqslant 3$ and $\tau \in\left\{\sigma_{1}, \bar{\sigma}_{1}, \ldots, \sigma_{9}, \bar{\sigma}_{9}\right\}$, the group $\Gamma(2 \pi / p, \tau)$ is contained in an arithmetic lattice in $\mathrm{SU}(2,1)$ if and only if $p=3$ and $\tau=\bar{\sigma}_{4}$.

We will use the following criterion for arithmeticity:

Proposition 4.1. Let $E$ be a purely imaginary quadratic extension of a totally real field $F$, and let $H$ be an $E$-defined Hermitian form of signature $(2,1)$ such that a sporadic group $\Gamma$ is contained in $\mathrm{SU}\left(H ; \mathrm{O}_{E}\right)$. Then $\Gamma$ is contained in an arithmetic lattice in $\mathrm{SU}(2,1)$ if and only if for all $\varphi \in \operatorname{Gal}(F)$ not inducing the identity on $\mathbb{Q}[\operatorname{Tr} \operatorname{Ad} \Gamma]$, the form ${ }^{\varphi} \mathrm{H}$ is definite.

This follows from [Mostow 1980, Lemma 4.1]. Hypotheses (1) and (3) of that lemma - that $\mathbb{Q}[\operatorname{Tr} \operatorname{Ad} \Gamma]$ is a totally real field, and that $\operatorname{Tr} \operatorname{Ad} \gamma$ is an algebraic integer for all $\gamma \in \Gamma$ - are verified by Propositions 2.2 and 3.2, using the special values of $\tau$ for sporadic groups.

We will prove Theorem 4.1 in several parts using this criterion. The first result follows the same lines as the corresponding one in [Parker and Paupert 2009]:

Proposition 4.2. The sporadic group $\Gamma(2 \pi / p, \tau)$ is not contained in an arithmetic lattice in $\mathrm{SU}(2,1)$, with the following possible exceptions: 
- $\tau=\sigma_{1}$ and $p=4$ or $p \geqslant 8$;

- $\tau=\sigma_{2}$ and 3 or 4 or 5 divides $p$;

- $\tau=\bar{\sigma}_{2}$ and $p=8,9,10,12,14,15,16,18$;

- $\tau=\bar{\sigma}_{4}$ and $p=3$ or $p \geqslant 7$;

- $\tau=\sigma_{5}$ and 5 divides $p$;

- $\tau=\sigma_{7}$ and 7 divides $p$.

Proof. We conjugate the generators and Hermitian form as in Proposition 2.2 so that their entries lie in the ring $\mathbb{Z}\left[\tau, \bar{\tau}, e^{ \pm i \psi}\right]$, and are therefore algebraic integers in the field $\mathbb{Q}\left[\tau, \bar{\tau}, e^{i \psi}\right]$. (Recall that $\psi=2 \pi / p$ in our cases.) We then find in each case a number field $E$ as in Proposition 4.1 containing $\mathbb{Q}\left[\tau, \bar{\tau}, e^{i \psi}\right]$, and a Galois conjugation of $E$ that acts nontrivially on $\mathbb{Q}[\operatorname{Tr} \operatorname{Ad} \Gamma]$ and sends the Hermitian form to another indefinite form. For the values of $\tau$ and $p$ that are not excluded above, we can use the same argument as in [Parker and Paupert 2009], namely, that one of the Galois conjugations of $E$ sends the parameter $\tau$ to another value for which we know that the Hermitian form is indefinite (from our description of the parameter space). This requires using a Galois conjugation fixing $e^{2 i \pi / p}$. The details:

- For $\tau=\sigma_{1}$ or $\bar{\sigma}_{1}$, let $E=\mathbb{Q}\left[e^{i \pi / 6}, e^{i \pi / 4}, e^{2 i \pi / p}\right]$. If $p$ is not divisible by 3 or 4 , $\sigma_{1}$ is sent to $\bar{\sigma}_{1}$ by the Galois conjugation that sends $e^{i \pi / 6}$ to $e^{-i \pi / 6}$, sends $e^{i \pi / 4}$ to $e^{-i \pi / 4}$, and fixes $e^{2 i \pi / p}$. The corresponding Hermitian form is indefinite for $p=3,4,5,6,7$. This works for $p=5$ or 7 , but for $p=3,4$ or 6 we need to find another Galois conjugation. For $p=3$ or 6 , sending $e^{i \pi / 6}$ to $e^{7 i \pi / 6}$ (and for compatibility $e^{i \pi / 4}$ to $e^{-i \pi / 4}$ ) fixes $e^{2 i \pi / 3}$ (respectively $e^{2 i \pi / 6}$ ) and sends $\sigma_{1}$ to $e^{4 i \pi / 3} \bar{\sigma}_{1}$, which is equivalent to $\bar{\sigma}_{1}$. These various Galois conjugations act nontrivially on $\operatorname{Re}\left(e^{-i \psi} \tau^{3}\right)=5 \cos \psi+5 \sqrt{2} \sin \psi$, which is in $\mathbb{Q}[\operatorname{Tr} \operatorname{Ad} \Gamma]$.

- For $\tau \in\left\{\sigma_{2}, \bar{\sigma}_{2}, \sigma_{3}, \bar{\sigma}_{3}\right\}$, let $E=\mathbb{Q}\left[e^{i \pi / 6}, e^{i \pi / 5}, e^{2 i \pi / p}\right]$. If $p$ is not divisible by 3 or 4 or 5 , the Galois conjugation that sends $e^{i \pi / 5}$ to $e^{3 i \pi / 5}$, sends $e^{i \pi / 6}$ to $e^{7 i \pi / 6}$ and fixes $e^{2 i \pi / p}$ is one that swaps $\sigma_{2}$ and $\sigma_{3}$, as well as $\bar{\sigma}_{2}$ and $\bar{\sigma}_{3}$. The Hermitian form corresponding to $\sigma_{2}$ and $\sigma_{3}$ is indefinite for all $p \geqslant 3$; for $\bar{\sigma}_{2}$ it is indefinite for $3 \leqslant p \leqslant 19$, and for $\bar{\sigma}_{3}$ it is indefinite for $3 \leqslant p \leqslant 6$. This Galois conjugation acts nontrivially on $|\tau|^{2}=2+2 \cos (\pi / 5)$ (respectively $2+2 \cos (3 \pi / 5)$ ), which is in $\mathbb{Q}[\operatorname{Tr} \operatorname{Ad} \Gamma]$.

If $p$ is not divisible by 2 or 3 , the Galois conjugation sending $e^{i \pi / 6}$ to $e^{-i \pi / 6}$ and fixing the 2 other generators of $E$ sends $\sigma_{2}$ to $\bar{\sigma}_{2}$. This works unless $p=$ $8,9,10,12,14,15,16,18$.

- For $\tau=\sigma_{4}$ or $\bar{\sigma}_{4}$, let $E=\mathbb{Q}\left[e^{2 i \pi / 7}, e^{2 i \pi / p}\right]$, which contains $i \sqrt{7}=\sigma_{4}-\bar{\sigma}_{4}$. If $p$ is not divisible by 7 , the Galois conjugation sending $e^{2 i \pi / 7}$ to $e^{-2 i \pi / 7}$ and fixing the other generator of $E$ sends $\sigma_{4}$ to $\bar{\sigma}_{4}$. The corresponding Hermitian 
form is indefinite for $p=4,5,6$. This Galois conjugation acts nontrivially on $8 \operatorname{Re}\left(e^{-i \psi} \tau^{3}\right)=20 \cos \psi+4 \sqrt{7} \sin \psi$, which is in $\mathbb{Q}[\operatorname{Tr} \operatorname{Ad} \Gamma]$.

- For $\tau \in\left\{\sigma_{5}, \bar{\sigma}_{5}, \sigma_{6}, \bar{\sigma}_{6}\right\}$, let $E=\mathbb{Q}\left[e^{i \pi / 9}, e^{2 i \pi / 5}, e^{2 i \pi / p}\right]$. If $p$ is not divisible by 5 , the Galois conjugation sending $e^{2 i \pi / 5}$ to $e^{4 i \pi / 5}$ and fixing the 2 other generators of $E$ sends $\sigma_{5}$ to $\sigma_{6}$, and $\bar{\sigma}_{5}$ to $\bar{\sigma}_{6}$. The Hermitian form corresponding to $\sigma_{5}$ and $\sigma_{6}$ is indefinite for all $p \geqslant 3$; for $\bar{\sigma}_{5}$ it is indefinite for $p=2,4$, and for $\bar{\sigma}_{6}$ it is indefinite for $4 \leqslant p \leqslant 29$. This Galois conjugation acts nontrivially on $\operatorname{Re} \tau^{3}=$ $11 / 2+11 \cos (2 \pi / 5)$ (respectively $11 / 2+11 \cos (4 \pi / 5)$ ), which is in $\mathbb{Q}[\operatorname{Tr} \operatorname{Ad} \Gamma]$.

If $p$ is not divisible by 3 , the Galois conjugation sending $e^{i \pi / 9}$ to $e^{-i \pi / 9}$ and fixing the 2 other generators of $E$ sends $\sigma_{6}$ to $\bar{\sigma}_{6}$. This works for $p=5$ (the only case where Proposition 2.3 doesn't tell us that $\Gamma\left(2 \pi / p, \sigma_{6}\right)$ and $\Gamma\left(2 \pi / p, \bar{\sigma}_{6}\right)$ are nondiscrete).

- For $\tau \in\left\{\sigma_{7}, \bar{\sigma}_{7}, \sigma_{8}, \bar{\sigma}_{8}, \sigma_{9}, \bar{\sigma}_{9}\right\}$, let $E=\mathbb{Q}\left[e^{i \pi / 9}, e^{2 i \pi / 7}, e^{2 i \pi / p}\right]$. If $p$ is not divisible by 7 , the Galois conjugation sending $e^{2 i \pi / 7}$ to $e^{6 i \pi / 7}$ and fixing the 2 other generators of $E$ sends $\sigma_{7}$ to $\sigma_{9}$ and $\sigma_{9}$ to $\sigma_{8}$, and $\bar{\sigma}_{7}$ to $\bar{\sigma}_{9}$ and $\bar{\sigma}_{9}$ to $\bar{\sigma}_{8}$. The Hermitian form corresponding to $\sigma_{7}, \bar{\sigma}_{8}$ and $\sigma_{9}$ is indefinite for all $p \geqslant 4$ (even 3 for $\sigma_{7}, \sigma_{9}$ ); for $\bar{\sigma}_{7}$ it is indefinite for $p=2$, for $\sigma_{8}$ it is indefinite for $4 \leqslant p \leqslant 41$, and for $\bar{\sigma}_{9}$ it is indefinite for $4 \leqslant p \leqslant 8$. This Galois conjugation acts nontrivially on $|\tau|^{4}+|\tau|^{2}-2 \operatorname{Re} \tau^{3}=3+2 \cos (2 \pi / 7)$ (respectively $3+2 \cos (4 \pi / 7)$ and $3+2 \cos (6 \pi / 7))$, which is in $\mathbb{Q}[\operatorname{Tr} \operatorname{Ad} \Gamma]$.

Finally, we know from Proposition 2.3 that, for $\tau \in\left\{\sigma_{3}, \bar{\sigma}_{3}, \sigma_{8}, \bar{\sigma}_{8}, \sigma_{9}, \bar{\sigma}_{9}\right\}$, $\Gamma(2 \pi / p, \tau)$ is nondiscrete for all $p$ and in particular is not contained in an arithmetic lattice in $\operatorname{SU}(2,1)$.

We then examine the remaining cases, where we must now take into account the effect of our various Galois conjugations on $\psi=e^{2 i \pi / p}$. In what follows, the number field $E$ is a cyclotomic field $\mathbb{Q}\left[e^{2 i \pi / r}\right]$; the Galois group of $E$ consists of the automorphisms $\varphi_{n}$ sending $e^{2 i \pi / r}$ to $e^{2 i n \pi / r}$ for $(n, r)=1$. The following criterion [Parker and Paupert 2009, Corollary 2.7] expresses the determinant $\kappa$ of the Hermitian matrix $H_{\tau}$ in a convenient way:

Lemma 4.1. When $\tau=e^{i \alpha}+e^{i \beta}+e^{-i \alpha-i \beta}$ and $\sin (\psi / 2) \geqslant 0$, the matrix $H_{\tau}$ has signature $(2,1)$ if and only if

$$
\kappa=8 \sin (3 \alpha / 2+\psi / 2) \sin (3 \beta / 2+\psi / 2) \sin (-3(\alpha+\beta) / 2+\psi / 2)<0 .
$$

Proposition 4.3. $\Gamma(2 \pi / p, \tau)$ is not contained in an arithmetic lattice in $\mathrm{SU}(2,1)$ if

- $\tau=\sigma_{1}$ and $p=4$ or $p \geqslant 8$

- $\tau=\sigma_{2}$ and 3 or 4 or 5 divides $p$;

- $\tau=\bar{\sigma}_{4}$ and $p \geqslant 7$; 
- $\tau=\sigma_{5}$ and 5 divides $p ;$ or

- $\tau=\sigma_{7}$ and 7 divides $p$.

Proof. In each case, find a Galois conjugation $\varphi$ acting nontrivially on $\mathbb{Q}[\operatorname{Tr} \operatorname{Ad} \Gamma]$ such that two of $\varphi\left(e^{3 i \alpha / 2}\right), \varphi\left(e^{3 i \beta / 2}\right)$, and $\varphi\left(e^{-3 i(\alpha+\beta) / 2}\right)$ lie in the open upper half of the unit circle, and the third in the open lower half (or, in the case of $\tau=\bar{\sigma}_{4}$, all three in the lower half). Then this property is stable, that is, if $\varphi(\psi)$ is small enough, adding $\varphi(\psi) / 2$ to each of the three angles will not change it, where we think of $\varphi$ as acting on angles. The details:

- As before, for $\tau=\sigma_{1}$ let $E=\mathbb{Q}\left[e^{i \pi / 6}, e^{i \pi / 5}, e^{2 i \pi / p}\right]$; we will use $\varphi \in \operatorname{Gal}(E)$ fixing $\sigma_{1}$ up to a cube root of unity $\left(\bmod _{\times} e^{ \pm 2 \pi i / 3}\right)$. In the notation of Lemma 4.1 , the corresponding triple $(3 \alpha / 2,3 \beta / 2,-3(\alpha+\beta) / 2)$ is $(\pi / 2, \pi / 8,-5 \pi / 8)$. We can get $\varphi_{n}\left(\sigma_{1}\right)=\sigma_{1} \bmod _{\times} e^{ \pm 2 \pi i / 3}$ by sending $e^{i \pi / 4}$ to $e^{ \pm i \pi / 4}$ and fixing $e^{i \pi / 6} \bmod _{\times} e^{ \pm 2 \pi i / 3}$, or by sending $e^{i \pi / 4}$ to $e^{ \pm 3 i \pi / 4}$ and $e^{i \pi / 6}$ to $e^{7 i \pi / 6}=-e^{i \pi / 6} \bmod _{\times} e^{ \pm 2 \pi i / 3}$. This means that $n$ is congruent to $(1$ or $-1 \bmod 8)$ and $(1$ or 5 or $9 \bmod 12)$ in the first case, and to $(3$ or $-3 \bmod 8)$ and $(3$ or 7 or $11 \bmod 12)$ in the second. We win if we can find such an $n$, coprime with $p$ and such that $n \pi / p<\pi / 2$, that is, $n \leqslant 2 p+1$ (this is the largest angle by which one can rotate the 3 points on the unit circle without any of them changing sides). The first few solutions to the above congruencies are $n=(1), 3,9,11,17,19,25,27,33,35,41$. Start with $n=3$; this works as long as 3 doesn't divide $p$ and $p \geqslant 7$. We check that $\varphi_{5}(\kappa)<0$ (and $\varphi_{5}(\sqrt{2}) \neq \sqrt{2}$ ) for $p=4$. Assume then that 3 divides $p$, and use $n=11$; this works as long as 11 doesn't divide $p$ and $p \geqslant 23$. This leaves $p=9,12,15,18,21$; we check that $n=5$ works for $p=9,18,21$, that $n=7$ works for $p=12$, and $n=11$ for $p=15$. Assume then that 33 divides $p$, and use $n=17$; this works as long as 17 doesn't divide $p$ and $p \geqslant 34$. This leaves $p=33$, where we check that $\varphi_{5}(\kappa)<0$. We then go on in this fashion (skipping solutions like 27 and 33, which are divisible by 3 ), assuming that $3 \times 11 \times 17$ divides $p$ and using $n=19$ and so on. In this fashion $p$ increases multiplicatively, whereas solutions to the above congruences increase additively; therefore such $n$ exist by a wider and wider margin. We conclude inductively that such an $n$ exists for $p$ large enough (and we have checked the few exceptions for small $p$ ).

- As previously, for $\tau=\sigma_{2}$ or $\sigma_{3}$ let $E=\mathbb{Q}\left[e^{i \pi / 6}, e^{i \pi / 5}, e^{2 i \pi / p}\right]$ and consider $\varphi \in \operatorname{Gal}(E)$ sending $e^{i \pi / 5}$ to $e^{3 i \pi / 5}$ and $e^{i \pi / 6}$ to $e^{7 i \pi / 6}=-e^{i \pi / 6}$. Then $\varphi$ swaps $\sigma_{2}$ and $\sigma_{3}$. With the notation of Lemma 4.1, the corresponding triples

$$
(3 \alpha / 2,3 \beta / 2,-3(\alpha+\beta) / 2)
$$

are

$$
\begin{array}{ll}
(\pi / 2, \pi / 20,-11 \pi / 20) & \text { when } \tau=\sigma_{2}, \\
(\pi / 2,7 \pi / 20,-17 \pi / 20) & \text { when } \tau=\sigma_{3} .
\end{array}
$$


Now when 3 or 4 or 5 divide $p, \varphi$ also acts on $e^{2 i \pi / p}$.

If 4 divides $p$, writing $p=4 k,\left(e^{2 i \pi / p}\right)^{k}=i=\left(e^{i \pi / 6}\right)^{3}$ is sent to $-i$, so $\varphi\left(e^{2 i \pi / p}\right)$ must be a $k$-th root of $-i$; in other words, $\omega_{k} \cdot e^{-i \pi / 2 k}$ for a $k$-th root of unity $\omega_{k}$. In fact, if 3 or 5 don't divide $p$, one can send $e^{2 i \pi / p}$ to any $\omega_{k} \cdot e^{-i \pi / 2 k}$, say with $\omega_{k}=e^{2 i \pi / k}$ (this gives a better bound on $p$ than 1 ). Then $\psi / 2$ is sent to $3 \psi / 2$ (because $-\pi / 2 k+2 \pi / k=3 \pi / 2 k$ ), and the argument works for $3 \pi / p<11 \pi / 20$ $(p \geqslant 6)$ when $\tau=\sigma_{3}$, and $3 \pi / p<17 \pi / 20(p \geqslant 4)$ when $\tau=\sigma_{2}$. There remain the cases where 5 divides $p$, as well as $\tau=\sigma_{3}$ and $p=4$. In the latter case one can check that $\varphi_{13}(\kappa)<0$, with $\varphi_{13}(\cos 3 \pi / 5) \neq \cos 3 \pi / 5$.

Now suppose that 5 divides $p$ but 3 or 4 do not, and write $p=5 k$. As above, one can send $e^{2 i \pi / p}$ to $e^{6 i \pi / p}$, and the same argument tells us that $\varphi(\kappa)<0$ for $p \geqslant 4$ when $\tau=\sigma_{2}$ and $p \geqslant 6$ when $\tau=\sigma_{3}$. When $p=5$ and $\tau=\sigma_{3}$, one can again check that $\varphi_{13}(\kappa)<0$ (with $\left.\varphi_{13}(\cos 3 \pi / 5) \neq \cos 3 \pi / 5\right)$.

If 3 divides $p$, we find $\varphi \in \operatorname{Gal}(E)$ as above; specifically, we require that $\varphi\left(e^{i \pi / 5}\right)=e^{3 i \pi / 5}$ or $e^{-3 i \pi / 5}$ and $\varphi\left(e^{i \pi / 6}\right)=e^{7 i \pi / 6}$ up to a cube root of unity, so that $\varphi$ swaps $\sigma_{2}$ and $\sigma_{3}$ (up to a cube root of unity). Such a $\varphi$ is realized as a $\varphi_{n}$ if (and only if) $n$ is congruent to (3 or $-3 \bmod 10)$ and ( 3 or 7 or $11 \bmod 12$ ). The values of such $n$ are 3, 7, 23, 27, 43, 47, ... Moreover, with the angle triples as above, $\varphi_{n}(\kappa)<0$ for $n \pi / p<17 \pi / 20$ (when $\tau=\sigma_{2}$ ) or $n \pi / p<\pi / 2$ (when $\tau=\sigma_{3}$ ). We may use $n=7$ as long as 7 doesn't divide $p$, which works for $p \geqslant 9$ when $\tau=\sigma_{2}$, and $p \geqslant 15$ when $\tau=\sigma_{3}$. We then check the cases $p=6$ and $\tau=\sigma_{2}$, as well as $p=6,9,12$ and $\tau=\sigma_{3}$. It turns out that $n=7$ works for all of these (renormalizing $7 \times 2 \pi / 6$ when $p=6$ as $2 \pi / 6$ ). Now if 7 also divides $p$, we use the next solution $n=23$, which works for $p \geqslant 47$ when $\tau=\sigma_{2}$, and $p \geqslant 28$ when $\tau=\sigma_{3}$, as long as 23 doesn't divide $p$. We check that $n=11$ works for $p=21$ for $\tau=\sigma_{2}, \sigma_{3}$ and $p=42$ for $\tau=\sigma_{2}$. One can then assume that $21 \times 23$ divides $p$, and so on. We conclude inductively as above.

- For $\tau=\bar{\sigma}_{4}, E$ is as before $\mathbb{Q}\left[e^{2 i \pi / 7}, e^{2 i \pi / p}\right]$, and $(3 \alpha / 2,3 \beta / 2,-3(\alpha+\beta) / 2)=$ $(-3 \pi / 7,-6 \pi / 7,9 \pi / 7)$. If 7 doesn't divide $p$, consider $\varphi \in \operatorname{Gal}(E)$ fixing $e^{2 i \pi / 7}$ and sending $e^{2 i \pi / p}$ to $e^{2 i n \pi / p}$ with $(n, p)=1$ and $1<n \leqslant 3 p / 7$ (this is possible as $p \geqslant 7)$. Then $n \pi / p \leqslant 3 \pi / 7$ as required.

If 7 divides $p$, say $p=7 k$, one can again fix $e^{2 i \pi / 7}$ and send $e^{2 i \pi / p}$ to a $k$-th root of itself; when $k \geqslant 3$, letting $\varphi\left(e^{2 i \pi / p}\right)=e^{2 i \pi(1 / k+1 / p)}$ works (that is, $\left.\varphi(\kappa)<0\right)$, because $\pi / k+\pi / p<3 \pi / 7$. There remain only the cases $p=7$, where one can check that $\varphi_{2}(\kappa)<0$ with $\varphi_{2}(\cos 2 \pi / 7) \neq \cos 2 \pi / 7$, and $p=14$, where one can check that $\varphi_{9}(\kappa)<0$ with $\varphi_{9}(\cos \pi / 7) \neq \cos \pi / 7$.

- As previously, for $\tau=\sigma_{5}$ or $\sigma_{6}$ let $E=\mathbb{Q}\left[e^{i \pi / 9}, e^{2 i \pi / 5}, e^{2 i \pi / p}\right]$ and consider $\varphi \in$ $\operatorname{Gal}(E)$ sending $e^{2 i \pi / 5}$ to $e^{4 i \pi / 5}$ and fixing $e^{i \pi / 9}$. Then $\varphi$ swaps $\sigma_{5}$ and $\sigma_{6}$. With the notation of Lemma 4.1 , the corresponding triples $(3 \alpha / 2,3 \beta / 2,-3(\alpha+\beta) / 2)$ are 
$(\pi / 3,13 \pi / 30,-23 \pi / 30)$ when $\varphi(\tau)=\sigma_{5}$, and $(\pi / 3,31 \pi / 30,-41 \pi / 30)$ when $\varphi(\tau)=\sigma_{6}$.

If 5 divides $p$, say $p=5 k, \varphi$ must send $e^{2 i \pi / p}$ to a $k$-th root of $e^{4 i \pi / 5}$, and one can choose any of these if 3 does not divide $p$, such as $e^{4 i \pi / 5 k}$. When $\tau=\sigma_{5}$, this works for $2 \pi / p \leqslant 17 \pi / 30$ (and $p \geqslant 4$ ), and when $\tau=\sigma_{6}$ for $2 \pi / p \leqslant 11 \pi / 30$ (and $p \geqslant 6$ ). When $p=5$ and $\tau=\sigma_{6}$, one can check that $\varphi_{4}(\kappa)<0$ (with $\left.\varphi_{4}(\sqrt{3} \sin (2 \pi / 5)) \neq \sqrt{3} \sin (2 \pi / 5)\right)$.

Now if 3 also divides $p$, we must look more closely at how $\varphi$ is defined above. Namely, such a $\varphi$ is a $\varphi_{n}$ if and only if $n$ is congruent to $2 \bmod 5$ and $1 \bmod 18$. The smallest such $n$ is 37 . However one can relax slightly the definition of $\varphi$ to allow $\varphi\left(e^{i \pi / 9}\right)=\omega_{3} e^{i \pi / 9}$ for any cubic root of unity $\omega_{3}$, as this does not affect $\tau$. The conditions are then that $n$ should be congruent to $(2 \bmod 5)$ and ( 1 or 7 or 13 mod 18). We can then use $n=7$, unless 7 divides $p$. In that case $\varphi_{7}$ would work for $7 \pi / p \leqslant 17 \pi / 30$ (with $p \geqslant 13$ ) when $\tau=\sigma_{6}$, and for $7 \pi / p \leqslant 11 \pi / 30$ (with $p \geqslant 20$ ) when $\tau=\sigma_{5}$. Since at this point 15 divides $p$, there remains only the case where $p=15$ and $\tau=\sigma_{6}$, in which case one can check that $\varphi_{11}(\kappa)<0$ with $\varphi_{11}(\cos (2 \pi / 15)) \neq \cos (2 \pi / 15)$.

Finally, if 7 also divides $p$ (at this point $p$ is divisible by 105), we can do the same thing. That is, we claim that there exists $n$ congruent to $(2 \bmod 5)$ and $(1$ or 7 or $13 \bmod 18$ ), coprime with $p$ and such that $n \pi / p \leqslant 11 \pi / 30$ (that is, $n \leqslant 11 p / 30$ ). For $p=105 k, n=37$ satisfies these conditions for $1 \leqslant k \leqslant 36$. After that, suppose that 37 divides $p$ and so on; we conclude inductively as above.

- As before, for $\tau=\sigma_{7}$ let $E=\mathbb{Q}\left[e^{i \pi / 9}, e^{2 i \pi / 7}, e^{2 i \pi / p}\right]$ and consider $\varphi_{n} \in \operatorname{Gal}(E)$ sending $e^{2 i \pi / 7}$ to $e^{6 i \pi / 7}$ (respectively $e^{-2 i \pi / 7}$ ) and fixing $e^{i \pi / 9}$ (up to a cube root of unity). This means that $n$ should be congruent to $(3$ respectively $-1 \bmod 7)$ and ( 1 or 7 or $13 \bmod 18$ ). Then $\varphi_{n}\left(\sigma_{7}\right)=\sigma_{9}$ (respectively $\sigma_{7}$ ), and the corresponding triple $(3 \alpha / 2,3 \beta / 2,-3(\alpha+\beta) / 2)$ is

$$
(\pi / 3,47 \pi / 42,-61 \pi / 42) \quad(\text { respectively }(\pi / 3,11 \pi / 42,-25 \pi / 42)) .
$$

With these values, $\varphi_{n}(\kappa)<0$ when $n \pi / p \leqslant 19 \pi / 42$ (respectively $n \pi / p \leqslant 25 \pi / 42$ ). The smallest such $n$ is 13, which works for $p \geqslant 22$ (as long as 13 doesn't divide $p$ ). It remains to check $p=7,14$ or 21 (here 7 is assumed to divide $p$ ): $n=5$ works when $p=7$ or 21 , and $n=11$ works when $p=14$. If 13 divides $p$, use the next solution $n=31$, and so on. We conclude inductively as above.

Lemma 4.2. For $\tau=\bar{\sigma}_{2}$ and $p=8,9,10,12,14,15,16,18, \Gamma(2 \pi / p, \tau)$ is not contained in an arithmetic lattice in $\mathrm{SU}(2,1)$.

Proof. For each of these values we find a Galois conjugation $\varphi_{n}$ of $E$ such that $\varphi_{n}(\kappa)<0$, where $\kappa=\operatorname{det} H_{\tau}$, and acting nontrivially on $\mathbb{Q}[\operatorname{Tr} \operatorname{Ad} \Gamma]$. For this last condition, it suffices to check that $\varphi_{n}(\cos 2 \pi / p) \neq \cos 2 \pi / p$ (this is true for 
all cases below, except $n=7$ and $p=8$, in which case $\left.\varphi_{7}(\cos \pi / 5) \neq \cos \pi / 5\right)$. The condition $\varphi_{n}(\kappa)<0$ can easily be checked, for instance numerically. We claim that the following $\varphi_{n}$ satisfy these conditions when $\tau=\bar{\sigma}_{2}: \varphi_{7}$ works for $p=8,9,10,12$, and $\varphi_{11}$ works for $p=14,15,16,18$.

\section{Commensurability}

In this section we compare the adjoint trace fields of our sporadic groups with those of the previously known lattices in SU $(2,1)$, namely the Picard and Mostow lattices (see [Deligne and Mostow 1986; Mostow 1980; 1986; Sauter 1990; Thurston 1998; Parker 2008a] for an overview). From the lists on [Mostow 1980, p. 251; Deligne and Mostow 1986, p. 86; Thurston 1998, pp. 548-549], we see that for these lattices $\Gamma, \mathbb{Q}[\operatorname{Tr} \operatorname{Ad} \Gamma]$ is always of the form $\mathbb{Q}[\cos 2 \pi / d]$, where

- $d=3,4,5,6,8,9,10,12,18$ for the arithmetic Picard lattices;

- $d=12,15,20,24$ for the nonarithmetic Picard lattices;

- $d=1,8,10,12,15,18$ for the arithmetic Mostow lattices;

- $d=12,15,18,20,24,30,42$ for the nonarithmetic Mostow lattices.

Moreover, only two nonarithmetic noncocompact lattices are known in $\mathrm{SU}(2,1)$, both with $d=12$.

Remark 5.1. The nonarithmetic Picard and Mostow lattices in $\operatorname{SU}(2,1)$ fall into at least 7 and at most 9 distinct commensurability classes.

Indeed there are 6 distinct adjoint trace fields ( $d=15$ and 30 give the same field), and for $d=12$ there are two classes, one cocompact and the other noncocompact. Also, there are a priori 15 examples, but Mostow [1986] and Sauter [1990] find commensurabilities among some of them. See [Parker 2008a] for more details.

Now we use the values from Proposition 3.3 to distinguish commensurability classes of sporadic groups, from each other and from the Picard and Mostow lattices. We will also use the fact that arithmeticity and cocompactness are commensurability invariants. We summarize the results from this section:

Theorem 5.2. For $p \geqslant 2$ and $\tau \in\left\{\sigma_{1}, \bar{\sigma}_{1}, \ldots, \sigma_{9}, \bar{\sigma}_{9}\right\}$, sporadic groups $\Gamma(2 \pi / p, \tau)$ are not commensurable to any Picard or Mostow lattice, except possibly when

- $p=2$ or 4 or 6 and $\tau$ is any sporadic value;

- $p=3$ and $\tau=\sigma_{7}$

- $p=5$ and $\tau$ or $\bar{\tau}=\sigma_{1}, \sigma_{2}$;

- $p=7$ and $\tau=\bar{\sigma}_{4}$

- $p=8$ and $\tau=\sigma_{1}$

- $p=10$ and $\tau=\sigma_{1}, \sigma_{2}, \bar{\sigma}_{2}$; 
- $p=12$ and $\tau=\sigma_{1}, \sigma_{7}$;

- $p=20$ and $\tau=\sigma_{1}, \sigma_{2}$;

- $p=24$ and $\tau=\sigma_{1}$.

The first observation follows simply from the order of the complex reflections in the group; that is, from the fact that $\mathbb{Q}[\operatorname{Tr} \operatorname{Ad} \Gamma(2 \pi / p, \tau)]$ contains $\cos 2 \pi / p$. The values of $p \geqslant 3$ that we rule out are the divisors of $12,15,18,20,24,30,42$.

Lemma 5.1. For $p \neq 2,3,4,5,6,7,8,9,10,12,14,15,18,20,21,24,30$, 42, the sporadic groups $\Gamma(2 \pi / p, \tau)$ are not commensurable to any Picard or Mostow lattice. Moreover, they fall into infinitely many distinct commensurability classes.

We then examine the remaining values of $p$, where we can rule out most cases except when $p=3,4$ or 6 :

Lemma 5.2. For values $p \in\{5,7,8,9,10,12,14,15,18,20,21,24,30,42\}$, the sporadic groups $\Gamma(2 \pi / p, \tau)$ are not commensurable to any Picard or Mostow lattice, except possibly when

- $p=5$ and $\tau$ or $\bar{\tau}=\sigma_{1}, \sigma_{2}$;

- $p=7$ and $\tau=\bar{\sigma}_{4}$;

- $p=8$ and $\tau=\sigma_{1}$

- $p=10$ and $\tau=\sigma_{1}, \sigma_{2}, \bar{\sigma}_{2}$;

- $p=12$ and $\tau=\sigma_{1}, \sigma_{7}$;

- $p=20$ and $\tau=\sigma_{1}, \sigma_{2} ;$ or

- $p=24$ and $\tau=\sigma_{1}$.

Proof. We use the values found for $\mathbb{Q}[\operatorname{Tr} \operatorname{Ad} \Gamma]$ in Section 3, listed in the table at the end of that section, as well as the following criterion.

Let $p \geqslant 3, p \neq 6$ and $d \in \mathbb{N}$. Then $\sin 2 \pi / p=\cos (p-4) \pi / 2 p$ is in $\mathbb{Q}[\cos 2 \pi / d]$ if and only if

- $p$ divides $d$ (if 4 divides $p$ );

- $2 p$ divides $d$ (if $p$ is even but not divisible by 4); and

- $4 p$ divides $d$ (if $p$ is odd).

This allows us to rule out the cases

- $p=7,9,14,15,18,21,30,42$ when $\tau$ or $\bar{\tau}=\sigma_{1}$;

- $p=7,8,9,12,14,15,18,21,24,30,42$ when $\tau$ or $\bar{\tau}=\sigma_{2}$ or $\sigma_{3}$;

- $p=5,8,9,10,12,14,15,18,20,21,24,30$ when $\tau$ or $\bar{\tau}=\sigma_{4}$;

- $p=5,7,8,9,10,12,14,15,18,20,21,24,30,42$ when $\tau$ or $\bar{\tau}=\sigma_{5}$ or $\sigma_{6}$;

- $p=5,7,8,9,10,14,15,18,20,21,24,30,42$ when $\tau$ or $\bar{\tau}=\sigma_{7}$. 
Lemma 5.3. $\Gamma\left(2 \pi / 3, \bar{\sigma}_{4}\right)$ is not commensurable to any Picard or Mostow lattice.

Proof. Recall that this is the only arithmetic sporadic group. $\mathbb{Q}\left[\operatorname{Tr} \operatorname{Ad} \Gamma\left(2 \pi / 3, \bar{\sigma}_{4}\right)\right]$ contains $\sqrt{21}$, which is not in $\mathbb{Q}[\cos 2 \pi / d]$ for $d=1,3,4,5,6,8,9,10,12,15,18$.

Lemma 5.4. The groups $\Gamma\left(2 \pi / 3, \sigma_{1}\right), \Gamma\left(2 \pi / 3, \bar{\sigma}_{1}\right), \Gamma\left(2 \pi / 3, \sigma_{5}\right), \Gamma\left(2 \pi / 5, \sigma_{3}\right)$ and $\Gamma\left(2 \pi / 5, \bar{\sigma}_{3}\right)$ are not commensurable to any Picard or Mostow lattice.

Proof. In the groups $\Gamma\left(2 \pi / 3, \sigma_{1}\right), \Gamma\left(2 \pi / 3, \bar{\sigma}_{1}\right), \Gamma\left(2 \pi / 5, \sigma_{3}\right)$ and $\Gamma\left(2 \pi / 5, \bar{\sigma}_{3}\right)$, $R_{1} R_{2}$ is parabolic [Parker and Paupert 2009], whereas $R_{2}\left(R_{1} J\right)^{5}$ is parabolic in $\Gamma\left(2 \pi / 3, \sigma_{5}\right)$ (details to appear in a forthcoming paper). It follows from Godement's compactness criterion that such a group cannot be commensurable to a cocompact lattice. Therefore it suffices to check that these groups are not commensurable to the noncocompact Picard and Mostow lattices, which both have adjoint trace field equal to $\mathbb{Q}[\cos 2 \pi / 12]$. Now for $\tau=\sigma_{1}$ or $\bar{\sigma}_{1}, \mathbb{Q}[\operatorname{Tr} \operatorname{Ad} \Gamma(2 \pi / 3, \tau)]$ contains $\sqrt{2} \sin 2 \pi / p=\sqrt{6} / 2$, which is not in $\mathbb{Q}[\cos 2 \pi / 12]$, and in the three other cases $\mathbb{Q}[\operatorname{Tr} \operatorname{Ad} \Gamma]$ contains $\cos 2 \pi / 5$, which is not in $\mathbb{Q}[\cos 2 \pi / 12]$ either.

Lemma 5.5. $\Gamma\left(2 \pi / 3, \bar{\sigma}_{1}\right), \Gamma\left(2 \pi / 3, \sigma_{2}\right)$ and $\Gamma\left(2 \pi / 3, \bar{\sigma}_{2}\right)$ are not discrete, and therefore not commensurable to any Picard or Mostow lattice.

Proof. In the first of these groups $R_{1}\left(R_{1} J\right)^{4}$ is elliptic of infinite order, and in the two others $R_{1}\left(R_{1} J\right)^{5}$ is elliptic of infinite order (details to appear).

\section{Acknowledgments}

The author would like to thank John Parker and Domingo Toledo for stimulating conversations concerning this work.

\section{References}

[Deligne and Mostow 1986] P. Deligne and G. D. Mostow, "Monodromy of hypergeometric functions and nonlattice integral monodromy”, Inst. Hautes Études Sci. Publ. Math. 63 (1986), 5-89. MR 88a:22023a Zbl 0615.22008

[Goldman 1999] W. M. Goldman, Complex hyperbolic geometry, Oxford University Press, New York, 1999. MR 2000g:32029 Zbl 0939.32024

[Maclachlan and Reid 2003] C. Maclachlan and A. W. Reid, The arithmetic of hyperbolic 3-manifolds, Graduate Texts in Math. 219, Springer, New York, 2003. MR 2004i:57021 Zbl 1025.57001

[McReynolds 2006] D. McReynolds, "Arithmetic lattices in SU $(n, 1)$ ", preprint, 2006, Available at http://www.ma.utexas.edu/users/dmcreyn/ComplexArithmeticI.pdf.

[Mostow 1980] G. D. Mostow, "On a remarkable class of polyhedra in complex hyperbolic space", Pacific J. Math. 86:1 (1980), 171-276. MR 82a:22011 Zbl 0456.22012

[Mostow 1986] G. D. Mostow, "Generalized Picard lattices arising from half-integral conditions", Inst. Hautes Études Sci. Publ. Math. 63 (1986), 91-106. MR 88a:22023b Zbl 0615.22009 
[Parker 2008a] J. R. Parker, "Complex hyperbolic lattices", preprint, Univeristy of Durham, 2008, Available at http://maths.dur.ac.uk/ dma0jrp/img/Lattices.pdf.

[Parker 2008b] J. R. Parker, "Unfaithful complex hyperbolic triangle groups, I: Involutions", Pacific J. Math. 238:1 (2008), 145-169. MR 2009h:20056 Zbl 1158.20023

[Parker and Paupert 2009] J. R. Parker and J. Paupert, "Unfaithful complex hyperbolic triangle groups, II: Higher order reflections”, Pacific J. Math. 239:2 (2009), 357-389. MR 2009h:20057 Zbl 1161.20046

[Pratoussevitch 2005] A. Pratoussevitch, "Traces in complex hyperbolic triangle groups", Geom. Dedicata 111 (2005), 159-185. MR 2006d:32036 Zbl 1115.32015

[Sauter 1990] J. K. Sauter, Jr., "Isomorphisms among monodromy groups and applications to lattices in PU(1, 2)”, Pacific J. Math. 146:2 (1990), 331-384. MR 92d:22016 Zbl 0759.22013

[Thurston 1998] W. P. Thurston, "Shapes of polyhedra and triangulations of the sphere", pp. 511549 in The Epstein birthday schrift, edited by I. Rivin et al., Geom. Topol. Monogr. 1, Geom. Topol. Publ., Coventry, 1998. MR 2000b:57026 Zbl 0931.57010

Received February 23, 2009.

JULIEN PAUPERT

DEPARTMENT OF MATHEMATICS

UNIVERSITY OF UTAH

155 SOUTH 1400 EAST

SALT LAKE CITY, UT 84112

United STATES

paupert@math.utah.edu 